\title{
Sleep Quality in a Case-control Subset of Trisomy 21 and Typically Developing Children in Riyadh, Kingdom of Saudi Arabia
}

\author{
May W Al-Khudhairy ${ }^{1}$, Farah Al Shami ${ }^{2}$, Anfal AlOtebe ${ }^{3}$, Ghadah Al Abdan ${ }^{4}$, Hana Al Ghwery ${ }^{5}$, Muntaha Al Zughaibi ${ }^{6}$, \\ Reem Al Abdan ${ }^{7}$, Samaher Al Quhtani ${ }^{8}$
}

\begin{abstract}
Aim: To identify sleep-domains as well as clinical features in children with trisomy 21 that may shed light on a possible sleep disturbance as compared with typically developing (TD) age-matched-controls.

Materials and methods: A convenient sample of 100 Saudi Arabian children between the ages of 3-12 were invited to participate from several schools and centers in Riyadh during February 2018 to April 2018. Fifty of these children had trisomy 21, and 50 were TD age-matched-controls. A clinical examination of participants' oral characteristics as well as the Wisconsin's 22 item Children's Sleep Habit Questionnaire (CSHQ) were utilized to help identify the impact of these variables on sleep quality.

Results: Sleep domains that harbored strong evidence in differences between the two groups were sleep anxiety, parasomnia, and sleepdisordered-breathing. The clinical features unique to trisomy 21 were Mallampati score, macroglossia, lip-incompetence, tongue-ridging, and wear-facets.

Conclusion: Clinical features and sleep quality coexist, providing better insight into the degree of sleep disturbance a child with trisomy 21 may have.

Clinical significance: Dental clinicians must consider such factors that preclude to poor sleep, which might place children with trisomy 21 at a further disadvantage despite the fact that their baseline is already at risk of being at such a stage.

Keywords: Children's sleep habits questionnaire, Obstructive sleep apnea, Parasomnia, Sleep anxiety, Sleep-disordered breathing, Trisomy 21. The Journal of Contemporary Dental Practice (2019): 10.5005/jp-journals-10024-2718
\end{abstract}

\section{INTRODUCTION}

Sleep is an essential pillar in human health, more so in children's growth. Any disruption in sleep physiology may lead to detrimental health effects. 1

Down syndrome is also known as T21 ranks, amongst the commonest of chromosomal conditions afflicting 1:792 live births. ${ }^{2}$ In Saudi Arabia, the prevalence of T21 is $6.6: 10,000 .^{3}$

Children with T21 are more likely to suffer from obstructive sleep apnea (OSA) than their age-matched counterparts; a recent study by Chamseddin et al. found that $90 \%$ of their sample $(n=95$ of total $n=$ 106) were sufferers of OSA. The severity of OSA was directly correlated to obesity that were heavier were more at risk of severe OSA. ${ }^{4}$

OSA is caused by complete or partial obstructions of the upper airway resulting in a reduction of the airflow. ${ }^{5,6}$ This, if left untreated, could lead to detrimental effects and a negative impact on the quality of life of these children given their preexisting intellectual disabilities and difficulties that they are already challenged with.

Sleep can be evaluated via multiple tools. Both polysomnography $(\mathrm{PSG})^{7}$ (overnight multi-parametric diagnostic tool assessing sleep) and/or actigraphy ${ }^{8}$ (portable sleep assessment tool) are the bar, in reliability and validity for assessing sleep behavior, their use is expensive, time-consuming and difficult when used on a wide scale. Sleep diaries are also an excellent evaluator; however, they require the parents and/or guardians disciplined compliance in logging for longer periods. Sleep-related questionnaires have shown promising results in a matter of their economic and time efficiencies, which sets them as the preferred tool used by researchers internationally in predicting most sleep disorders. ${ }^{9}$ \begin{tabular}{l}
\hline \hline${ }^{1-8}$ Department of Oral and Maxillofacial Surgery and Diagnostic \\
Sciences, Riyadh Elm University, Riyadh, Kingdom of Saudi Arabia \\
Corresponding Author: May W Al-Khudhairy, Department of Oral and \\
Maxillofacial Surgery and Diagnostic Sciences, Riyadh Elm University, \\
Riyadh, Kingdom of Saudi Arabia, Phone: +966503476722 , e-mail: \\
may.alkhudhairy@riyadh.edu.sa
\end{tabular}

How to cite this article: Al-Khudhairy MW, Al Shami F, AlOtebe A, et al. Sleep Quality in a Case-control Subset of Trisomy 21 and Typically Developing Children in Riyadh, Kingdom of Saudi Arabia. J Contemp Dent Pract 2019;20(12):1424-1429.

Source of support: Nil

Conflict of interest: None

A study by Markovich et al. validated the CSHQ against both PSG and actigraphy. The study was conducted on 30 children aged between 6 years and 12 years of age with no known sleep disorders. They used the 33 items CSHQ, which was further analyzed into eight subscales; bedtime resistance, sleep onset delay, sleep duration, sleep anxiety, night waking, parasomnias, sleep-disordered breathing (SDB), and daytime sleepiness. ${ }^{10}$

The CSHQ, according to Owens et al., is an effective sleep screening tool that can discern both behaviorally-based as well as medically based disturbed sleep in school-aged children. ${ }^{11}$

There was a recent study that proposed a model for those with T21 to screen for OSA that did not need to have a sleep study, via urine markers, imaging, and physical examination. ${ }^{12}$

Sleep is crucial to well-being, more so to children where lack of sleep is linearly correlated to its adverse effects. Today's sleep hours

(0) The Author(s). 2019 Open Access This article is distributed under the terms of the Creative Commons Attribution 4.0 International License (https://creativecommons. org/licenses/by-nc/4.0/), which permits unrestricted use, distribution, and non-commercial reproduction in any medium, provided you give appropriate credit to the original author(s) and the source, provide a link to the Creative Commons license, and indicate if changes were made. The Creative Commons Public Domain Dedication waiver (http://creativecommons.org/publicdomain/zero/1.0/) applies to the data made available in this article, unless otherwise stated. 
have been constantly deferred, without any change in morning hours culminating to a collective decrease in average sleep hours, which has, in turn, led to distinctive discord in children's behavior including and not limited to attention deficit. ${ }^{13}$

It was discovered that children were more prone to sadness, anger, and shortcoming in attention, ensuing from decreased hours of sleep. Furthermore, following research involved in controlled sleep deprivation, results elicited serious deleterious effects in children's management of their emotions, cloudy memory, and poorer academic conduct. ${ }^{\text {? }}$

The aim of this study was to target the sleep domains from the Wisconsin's Children's Sleep Habit Questionnaire (W-CSHQ) acting as possible risk factors in sleep quality in children with T21, with a sample of TD age-matched controls. In addition, to investigate the clinically related factors correlating with disturbed sleep.

\section{Materials and Methods}

\section{Study Population}

All Saudi Arabian nationals with T21 and TD aged between 3 and 12 were included in the study. Those patients that had GERD, thyroid disorders, asthma, hearing and/or a visual impairment, major psychiatric illness, maxillofacial deformities, and/or any prescribed medication were excluded from the study. The participants recruited were of a convenient, readily available population in the capital city of Riyadh from various local schools and centers from the periods of February 2018 to April 2018. History was taken from all study participants, aid in the completion of questionnaire as well as a thorough clinical examination.

The Institutional Review Board of Riyadh Elm University (formerly known as Riyadh Colleges of Dentistry and Pharmacy) evaluated the study protocol following its registration at the Research Center (FRP/2018/64) and approved it, as it was compliant with Declaration of Helsinki and designated approval \# RC/ IRB/2016/708. Informed consent was obtained from the guardians of children abiding by the laws dictated by the Declaration of Helsinki.

The examiners prior to commencing the study conducted a pilot for inter-and intra-examiner reliability pertinent to the clinical examination trajectory of the study.

\section{Questionnaire Completion, Measurement of Anthropometric markers and Clinical Examination}

We used the 22 items W-CSHQ, translated into the Arabic language. ${ }^{14}$ The 22 questions belong to one of the established 8 sleep-related domains; bedtime resistance, sleep onset delay, sleep duration, sleep anxiety, night wakings, parasomnias, SDB, and daytime sleepiness.

1. Child goes to bed at the same time at night

2. Child falls asleep within 20 minutes after going to bed.

3. Child falls asleep in own bed

4. Child falls asleep in parents' or siblings' bed

5. Child falls asleep with rocking or rhythmic movements

6. Child needs special object to fall asleep

7. Child needs parent in the room to fall asleep

8. Child resists going to bed at bedtime

9. Child is afraid of sleeping in the dark

10. Child sleeps about the same amount each day

11. Child is restless and moves a lot during sleep

12. Child moves to someone else's bed during the night

13. Child grinds teeth during sleep
14. Child snores loudly

15. Child awakens during the night and is sweating screaming and inconsolable

16. Child naps during the day

17. Child wakes up once during the night

18. Child wakes up more than once during the night

19. Child wakes up by him/herself

20. Child wakes up very early in the morning

21. Child seems tired during the daytime

22. Child falls asleep while involved in activities

The eight domains are:

1. Bedtime resistance: Refers to the refusal of a child going to bed via different behaviors, all leading to shorter sleep duration.

2. Sleep onset delay: Meaning that the child did not fall asleep within 20 minutes of supposed bedtime.

3. Sleep duration: Refers to what a parent and/or guardian assume is an adequate length of time that a child gets in means of sleep and whether it is consistent every evening.

4. Sleep anxiety: The amount of negative or fearful thoughts, prior to bedtime, at home or away from home, ultimately detrimental to restful sleep.

5. Night wakings: The measure of how often a child awakened through the night, whether the child moved to a sibling(s) or parents' bed, how disturbing it is to the child's sleep quality.

6. Parasomnias: Sleep-related undesirable unconscious nocturnal behaviors including and not limited to sleep talking, enuresis and sleep bruxism (SB).

7. Sleep-disordered breathing: Related to all variables leading to SDB, be it snoring, temporary cessation of breathing, snorting as well as gasping. Sleep-disordered breathing is a lay term to obstructive sleep apnea, which ultimately is an abnormal breathing pattern.

8. Daytime sleepiness: Pertains to how the parent and or guardian views the child's sleepiness due to being tired during the day.

The W-CSHQ was sent home to be completed by the parents and/ or guardians of the children and was later collected from the schools and centers. Those that completed the forms and provided their consent were the ones whose children were enrolled in the study.

Anthropometric measurements as well as an extraoral and intraoral clinical examination then followed which included age, gender, race, body mass index, blood pressure, oxygen saturation by use of pulse oximeter, neck circumference, Mallampati score (class I: soft palate, uvula, fauces, pillars visible, class II: soft palate, major part of uvula, fauces visible, class III: soft palate, base of uvula visible, class IV: only hard palate visible). Dental examination included dental classification, overjet recorded in millimeters, overbite recorded as a percentage, presence of wear facets, tongue ridging, and subjective presence of macroglossia.

Interexaminer reliability was evaluated in a subset of 10-piloted TD controls whereby interexaminer reliability was established by two trained examiners (F.A and S.A): both scored their clinical evaluations. The second examiner was blinded to the first examiner's scoring. Test-retest reliability was assessed by conducting a second examination with the same TD participant(s) and evaluation within 1 week after the initial clinical examination.

\section{Statistical analysis}

The study was subjected to Statistical Package for Social Sciences software (SPSS, version 22, SPSS Inc., Chicago, IL, USA). A Fisher's exact 
test was performed to analyze the difference in the proportion of "Yes" responses and for each "Yes" and "No" question. The control and clinical samples were compared using an analysis of covariance (ANCOVA). Chi-square test with a $5 \%$ level of significance was used for categorical data. Student $t$ test was used for quantitative data between groups. Data are presented as mean \pm standard deviation, and $p<0.05$ was considered statistically significant. The power of the sample was 0.8 analyzed at a significance level of 0.05 at which statistical power was found to be satisfactory ( $80 \%)$ for detecting the effects of medium size.

\section{Results}

A total of 100 patients from an original for 128 were recruited for this study; 50 TD controls and 50 T21 were included in analyses, achieving our targeted sample size. Both TD and T21 groups had equal number of males and females. The demographical data, anthropometrical data, and results from physical examination of TD and T21 children are shown in Tables 1 to 3.

The mean (SD) height of the TD participants showed strong evidence as higher than the T21 group ( $p<0.05)$. The mean (SD) weight of the TD controls was also higher than the T21 group; however, it showed weak evidence $(p>0.05)$. There was weak evidence in any difference in the body mass index between the TD controls and T21 group ( $p>0.05$ ). The mean (SD) oximeter of the T21 group was higher than TD controls, but it was weak evidence $(p>0.05)$. Both the mean (SD) systolic and diastolic blood pressure of T21 group showed strong evidence of being lower than TD control ( $p<0.05$ ). The mean (SD) neck circumference of the T21 Group was higher than TD controls. However, it showed weak evidence $(p>0.05)$ in this particular sample.

The average overbite and mean (SD) overjet among TD were higher than T21 children. However, the difference showed weak evidence $(p>0.05)$ (Table 2). T21 children were more likely to have macroglossia than TD, and this association harbored strong evidence $(p<0.05)$. Furthermore, there was strong evidence to show that T21 children were more likely to have Mallampati

Table 1: Demographic and clinical data of typically developing controls and trisomy 21 children

\begin{tabular}{|c|c|c|c|c|}
\hline & & $\begin{array}{l}\text { Typically } \\
\text { developing con- } \\
\text { trols mean (SD) }\end{array}$ & $\begin{array}{l}\text { Trisomy } 21 \\
\text { mean (SD) }\end{array}$ & $p$ value \\
\hline \multicolumn{2}{|l|}{ Age } & $8.02(1.78)$ & $7.52(2.88)$ & 0.474 \\
\hline \multicolumn{2}{|l|}{ Height (cm) } & $126.58(12.45)$ & $115.64(14.26)$ & $0.001^{*}$ \\
\hline \multicolumn{2}{|l|}{ Weight (kg) } & 28.78 (10.65) & 25.52 (10.79) & 0.091 \\
\hline \multicolumn{2}{|l|}{ Body mass index } & $17.82(4.26)$ & $17.93(4.84)$ & 0.452 \\
\hline \multicolumn{2}{|l|}{ Oximeter } & $94.54(5.6)$ & $96.04(5.14)$ & 0.203 \\
\hline \multicolumn{2}{|l|}{ Pulse } & $94.72(18.05)$ & $92.98(19.42)$ & 0.444 \\
\hline \multirow[t]{2}{*}{ Blood pressure } & Systolic & $115.26(27.34)$ & $101.92(23.36)$ & $0.016^{*}$ \\
\hline & Diastolic & 78.68 (23.36) & $66.54(15.19)$ & $0.002^{*}$ \\
\hline \multicolumn{2}{|c|}{ Neck circumference (cm) } & $28.42(2.54)$ & $29.78(4.53)$ & 0.180 \\
\hline
\end{tabular}

*Statistically significant

Table 2: Overbite and overjet of typically developing controls and trisomy 21 children

\begin{tabular}{lcll}
\hline & $\begin{array}{l}\text { Typically developing } \\
\text { controls }\end{array}$ & Trisomy 21 & p value \\
\hline Overbite $(\%)$ & $19.40(26.83)$ & $9.60(24.66)$ & 0.085 \\
Overjet $(\mathrm{mm})$ & $2.28(1.69)$ & $1.32(1.83)$ & 0.059 \\
\hline
\end{tabular}

Table 3: Dental examination of typically developing controls and trisomy 21 children

\begin{tabular}{|c|c|c|c|c|}
\hline & & \multicolumn{2}{|c|}{ Frequency (percent) } & \multirow[b]{2}{*}{$p$ value } \\
\hline & & $\begin{array}{l}\text { Typically } \\
\text { developing } \\
\text { controls }\end{array}$ & Trisomy 21 & \\
\hline \multirow[t]{2}{*}{ Macroglossia } & Yes & $2(4.0)$ & $37(74.0)$ & $0.000^{*}$ \\
\hline & No & $48(96.0)$ & $13(26.0)$ & \\
\hline \multirow[t]{4}{*}{ Mallampati score } & I & $25(50.0)$ & $1(2.0)$ & $0.000^{*}$ \\
\hline & ॥ & $22(44.0)$ & $10(20.0)$ & \\
\hline & III & $3(6.0)$ & $19(38.0)$ & \\
\hline & IV & $0(0.0)$ & $20(40.0)$ & \\
\hline \multirow[t]{3}{*}{ Dental classification } & Class I & $23(46.0)$ & $16(32.0)$ & 0.132 \\
\hline & Class II & $19(38.0)$ & $18(36.0)$ & \\
\hline & Class III & $8(16.0)$ & $16(32.0)$ & \\
\hline \multirow[t]{2}{*}{ Tongue ridging } & Yes & $2(4.0)$ & $20(40.0)$ & $0.000^{*}$ \\
\hline & No & $48(96.0)$ & $30(60.0)$ & \\
\hline \multirow[t]{2}{*}{ Wear facets } & Yes & $2(4.0)$ & $20(40.0)$ & $0.000^{*}$ \\
\hline & No & $48(96.0)$ & $30(60.0)$ & \\
\hline \multirow[t]{2}{*}{ Lip competence } & Yes & $41(82.0)$ & $16(32.0)$ & $0.000^{*}$ \\
\hline & No & $9(18.0)$ & $34(68.0)$ & \\
\hline \multirow[t]{2}{*}{ Asthma } & Yes & $2(4.0)$ & $5(10.0)$ & 0.436 \\
\hline & No & $48(96.0)$ & $45(95.0)$ & \\
\hline
\end{tabular}

*Statistically significant

score IV and TD more likely to have Mallampati score I $(p<0.05)$. There was not strong evidence between T21 children who were more likely to have class III dental classification, and TD were more likely to have class I ( $p>0.05)$.

There was strong evidence showing T21 children were more likely to have tongue ridging and wear facets than TD controls $(p<0.05)$. Also, T21 children to have lip incompetence than TD who had lip competence $(p<0.05)$. Although, there was weak evidence in T21 children's likelihood to suffer from asthma compared to TD control $(p>0.05)$. T21 children were more likely to have poor, and TD controls were more likely to have moderate CSHQ. However, this was weak in evidence ( $p>0.05)$ (Fig. 1).

Table 4 the mean (SD) score on the W-CSHQ of TD controls was lower than T21 children for the following: "child needs parent in the room to fall asleep", "child is restless and moves a lot during sleep," and "child snores loudly" ( $p<0.05)$ and it harbored strong evidence.

\section{Discussion}

A study by Maris et al. was conducted to evaluate the severity of OSA retrospectively in $n=122$ boys age ranging from 4 years to 18 years. There was OSA in $66 \%$ of the sample, and nearly half of them had severe OSA. ${ }^{15}$

Similarly, a recent study showed that T21 was surprisingly at the lowest odds risk for depression when compared to other forms of intellectual disabilities such as autism and attention deficit hyperactivity disorder. ${ }^{16}$

Alarmingly, a study on T21 by Dekker et al. on early onset of Alzheimer's disease (AD) showed that anxiety and increased sleep disturbances, serve as a red flag, for an incidence of attention deficit in T21. This study included and was not limited to participants over the age of 30, which differs from our target age group, however, accentuates the degree of importance of sleep quality on T21 overall prospective quality of life. ${ }^{17}$ 


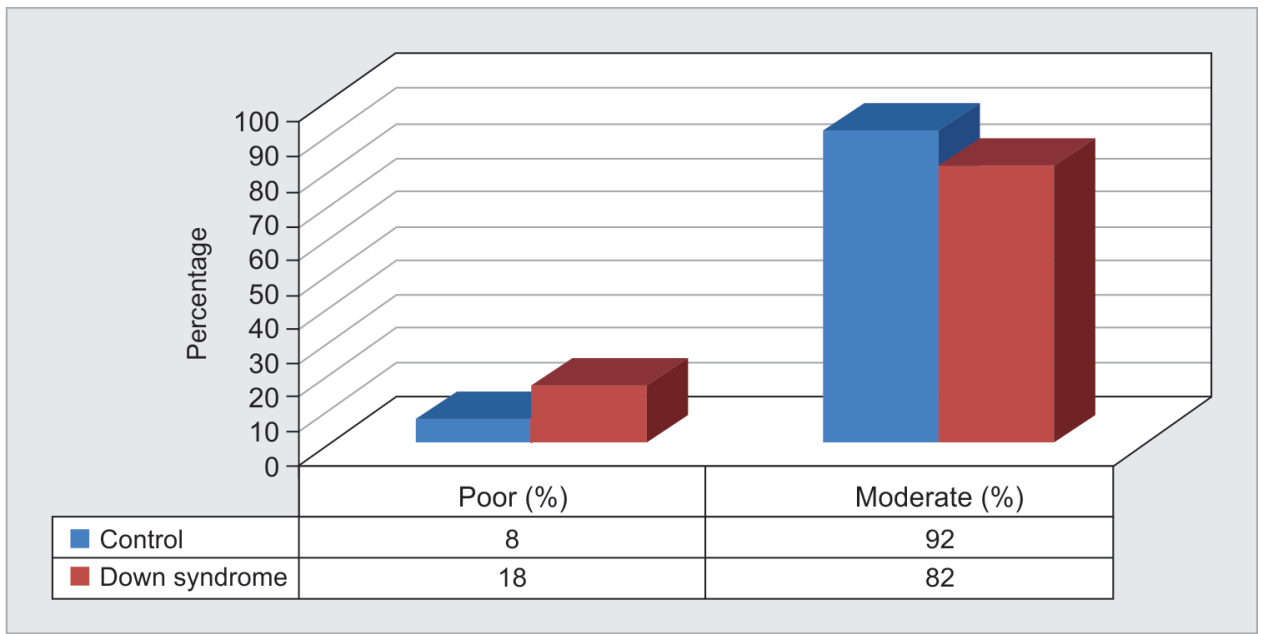

Fig. 1: Children's sleep habit questionnaire of typically developing controls and trisomy 21 children

A retrospective study by Esbensen et al. on around a 1,000 children with T21 age ranged from 5 years to 21 years showed the medical records including sleep studies indicative for OSA and questionnaires on behavioral sleep disorder were lacking $(3.5 \%$ and $8.6 \%$, respectively for OSA and behavioral sleep disorder) which is a medical tragedy since this population is in dire need of tools that are valid and reliable in extrapolating imperative information that helps determine quality of life of a population that is already at a disadvantage. ${ }^{18}$

Table 4: Mean (SD) score of 22 item Wisconsin's children's sleep habit questionnaire in each of typically developing controls and trisomy 21 children

\begin{tabular}{|c|c|c|c|c|c|}
\hline \multirow[b]{2}{*}{ Variables } & \multicolumn{2}{|c|}{$\begin{array}{c}\text { Typically developing } \\
\text { controls }\end{array}$} & \multicolumn{2}{|c|}{ Trisomy 21} & \multirow[b]{2}{*}{$p$ value } \\
\hline & Mean & $S D$ & Mean & $S D$ & \\
\hline 1 & 0.7600 & 0.43142 & 0.7400 & 0.44309 & 0.818 \\
\hline 2 & 0.7000 & 0.46291 & 0.6600 & 0.47852 & 0.670 \\
\hline 3 & 0.8200 & 0.38809 & 0.8400 & 0.37033 & 0.791 \\
\hline 4 & 0.7400 & 0.44309 & 0.8000 & 0.40406 & 0.478 \\
\hline 5 & 0.8800 & 0.32826 & 0.8800 & 0.32826 & 1.000 \\
\hline 6 & 0.7400 & 0.44309 & 0.8000 & 0.40406 & 0.478 \\
\hline 7 & 0.3400 & 0.47852 & 0.6400 & 0.48487 & $0.003^{*}$ \\
\hline 8 & 0.5400 & 0.50346 & 0.5400 & 0.50346 & 1.000 \\
\hline 9 & 0.5600 & 0.50143 & 0.6800 & 0.47121 & 0.219 \\
\hline 10 & 0.7800 & 0.41845 & 0.8400 & 0.37033 & 0.447 \\
\hline 11 & 0.5400 & 0.50346 & 0.8000 & 0.40406 & $0.006^{*}$ \\
\hline 12 & 0.6000 & 0.49487 & 0.7400 & 0.44309 & 0.139 \\
\hline 13 & 0.7800 & 0.41845 & 0.8800 & 0.32826 & 0.185 \\
\hline 14 & 0.5800 & 0.49857 & 0.8800 & 0.32826 & $0.001^{*}$ \\
\hline 15 & 0.8800 & 0.32826 & 0.9600 & 0.19795 & 0.142 \\
\hline 16 & 0.5600 & 0.50143 & 0.6200 & 0.49031 & 0.544 \\
\hline 17 & 0.4800 & 0.50467 & 0.6200 & 0.49031 & 0.162 \\
\hline 18 & 0.7800 & 0.41845 & 0.9000 & 0.30305 & 0.103 \\
\hline 19 & 0.7600 & 0.43142 & 0.6800 & 0.47121 & 0.375 \\
\hline 20 & 0.6200 & 0.49031 & 0.6800 & 0.47121 & 0.531 \\
\hline 21 & 0.6200 & 0.49031 & 0.7800 & 0.41845 & 0.082 \\
\hline 22 & 0.8400 & 0.37033 & 0.8800 & 0.32826 & 0.566 \\
\hline
\end{tabular}

Patients can report bruxism, especially if it is nocturnal clenching (tonic bruxism) behavior and witnessed clinically as wear facets and tongue ridging in cases of phasic bruxism, also awake bruxism. T21 belongs to the family of hyperkinetic movement disorders such as, autism and in a review article by Ella et al., the incidence of awake bruxism in a T21 population was a respectable $42 \%$, which is influenced by anxiety and indirectly by sleep quality. ${ }^{19}$ Our study showed clinical parameters of tongue ridging and wear facets that showed strong evidence in T21 than the TD agedmatched controls.

An interesting study by Churchill et al. reported the relationship between children of T21 sleep problems and the implication it had on health and function or accomplishment it had on everyday life as compared with TD age-matched controls. Hence, they looked at the eight domains of sleep disturbance markers and their effect on daily life habits. Sleep-disordered breathing, parasomnia, and sleep anxiety all had a negative impact on T21 children's ability to accomplish day-to-day activities. Sleep anxiety and parasomnias affected the T21 fitness score negatively, and it was significantly different from TD children. Sleep-disordered breathing was a significant explanatory factor for fitness, personal care, home life, mobility, responsibilities, school, and recreation. Findings of this study highlight the effect of disturbed sleep negative association with the accomplishment of daily activities. ${ }^{20}$ It is more than a coincidence that our study sheds light on the same domains affected by Churchill's study, which are sleep anxiety, parasomnia, and SDB. In addition, children without T21 display similar sleep disturbance, but those with T21 have an exaggerated effect due to their underlying developmental delay, which keeps them at a handicap.

To reiterate, the three domains that showed strong evidence in differences in TD controls and affected by T21 condition in our study included sleep anxiety (question 7), parasomnia (question 11), and SDB (question 14). Similarly, the clinical features harboring strong evidence and being unique in T21 children in our study are those with a high Mallampati, score, macroglossia, lip incompetence, tongue ridging, and wear facets; which are in effect the main causes for deterioration of sleep quality.

A recent cross-sectional study in South Korea ${ }^{22}$ sheds light on the male gender, older age, and greater developmental delay as the cardinal risk factors for sleep problems in children with T21. Although our sample had a younger age group than the South 
Korean study, there does seem to be a vicious cycle involved if sleep problems begin and are unaddressed then they will lead to daytime somnolence that affects learning and will further exacerbate the developmental delay, which in turn affects the sleep quality. As was mentioned earlier, children with T21 are at a disadvantage with their wider neck circumference and macroglossia, which is already a risk for OSA, the latter of which was significantly related in our study. Higher Mallampati score is seen in higher body mass index and was also seen in our study, factor in poor sleep quality, and this would work synergistically and catastrophically adding fuel to the ongoing fire. Strangely, obesity does not play a role on an increased incidence of OSA in T21 children as it does in the general population and TD children. As a matter of fact, our findings of higher Mallampati score and macroglossia are in fact in accordance with the South Korean study and a prior study conducted in Japan where certain anatomical factors including and not limited to tonsillar and adenoidal hypertrophy, macroglossia and hypotonia were more relevant markers of parent-reported sleep problems than a higher body mass index. ${ }^{22,23}$ Additionally, lip incompetence suggests a sign of mouth breathing to overcome the OSA. Both the tongue ridging and wear facets forewarn of $\mathrm{SB}$, which is exacerbated in a sleep condition, as is seen in our sample. Sleep bruxism is when a subject grinds or clenches their teeth nocturnally. It is known that a dental advancement appliance shows remarkable improvement in SB activity. That is, when sleep is disturbed, as is the case in OSA, there is an increased activity of SB. Sleep bruxism is asked of parents/ guardians in the 22 items W-CSHQ and is considered a parasomnia, ironically it is a subconscious defense mechanism of the body against OSA, as it reestablishes airway patency nocturnally. ${ }^{24}$

Treatment options include and are not limited to prophylactic and treatment, the former with good evaluations and monitoring. The latter option follows a definitive diagnosis of OSA via PSG to determine the level of OSA, mild, moderate, or severe. In cases of mild to moderate OSA, treatments range from a regiment of intranasal steroids to more time committed patient compliant palatal expansion. Surgical options vary from adenoidectomy, tonsillectomy, and upper palatopharyngoplasty, all considering that the benefit outweighs the disadvantage of placing a T21 child under general anesthesia. A dental sleep advancement appliance for mild to moderate OSA in adult T21 cases as per the guidelines of the American Academy of Sleep Medicine would be suitable. However, in cases of severe OSA, a continuous positive airway pressure machine would be the gold standard of treatment for both child and adult T21 subjects. ${ }^{25}$

Therefore, good sleep hygiene and early detection via questionnaires and clinical examination can identify those at risk at an early age. This will help not only identify those at risk but also monitor their sleep quality and their developments both physically and academically. Livability and lifestyle of children with T21 can be improved via a protocol of early clinical marker detection.

The limitation of the present study, in brief, are amongst and not limited to the size of the sample, as well as the convenient samples for both of the T21 and TD controls. In spite of the limitations, one could build on these impediments to improve future research considerations.

\section{Conclusion}

Our study extrapolated clinical findings in line with the sleep domains different between T21 and TD children. T21 are born with their underlying congenital disadvantage. Most importantly, could the mitigation of children with T21 sleep disorders help improve sleep, which in turn helps them improve their day-to-day accomplishments? What a service one can perform if extrapolating these simple easy clinical markers to help improve quality of sleep and in turn, quality of life in these so special of needs children!

A recent study on mothers' perspectives of children with T21 and social activities in the Kingdom of Saudi Arabia sadly deciphered a key shackle or impediment into their children's activity. ${ }^{21}$ The main way to overcome such obstacles was the modification of their environment. All roads lead to Rome, sleep being Rome in this case. The most efficient way is by ensuring a good night's sleep!

\section{Clinical Significance}

Not only an early recognition but identification of sleep impairment in T21 children, a vulnerable population, could in turn, improve cognition and an advantage early in life to learn a new skill that can foster a sense of independence.

\section{Acknowledgments}

The authors would like to acknowledge the assistance of the children and their parents and guardians for their consent to participate in this commendable study that sheds light on the many challenges faced by T21 children, not to mention the sleep disorder aspect. Authors would also like to offer their sincere appreciation to the centers that accommodated their crew and facilitated this research to be conducted.

\section{References}

1. Prado IM, Abreu LG, Silveira KS, et al. Study of associated factors with probable sleep bruxism among adolescents. J Clin Sleep Med 2018;14(8):1369-1376. DOI: 10.5664/jcsm.7276.

2. De Graaf G, Buckley F, Skotko BG. Estimates of Live Births, Natural Losses, and Elective Terminations with Down Syndrome in the United States. Am J Med Genet A 2015;167(4):756-767. DOI: 10.1002/ ajmg.a.37001.

3. Al Salloum A, El Mouzan MI, Al Herbish A, et al. Prevalence of selected congenital anomalies in Saudi Children: a community based study. Ann Saudi Med 2015;35(2):107-110. DOI: 10.5144/0256-4947.2015.107.

4. Chamseddin BH, Johnson RF, Mitchell RB. Obstructive sleep apnea in children with down syndrome: demographic, clinical and polysomnographic features. Otolaryngol Head Neck Surg 2019;160(1):150-157. DOI: 10.1177/0194599818797308.

5. Guilleminault C, Tilkian A, Dement WC. The sleep apnea syndromes. Annu Rev Med 1976;27:465-484. DOI: 10.1146/annurev. me.27.020176.002341.

6. Guilleminault C, Quo SD. Sleep disordered breathing. a view at the beginning of the Millennium. Dent Clin North Am 2001;45(4):643-656.

7. Chung F, Yegneswaran B, Liao $P$, et al. Validation of the Berlin questionnaire and American Society of Anethesiolo-gists checklist as screening tools for obstructive sleep apnea in surgical patients. Anesthesiology 2008;108(5):822-830. DOI: 10.1097/ ALN.0b013e31816d91b5.

8. Meltzer LJ, Walsh CM, Traylor J, et al. Direct comparison of two new actigraphs and polysomnography in children and adolescents. Sleep 2012;35(1):159-166. DOI: 10.5665/sleep.1608.

9. Chervin RD, Weatherly RA, Garetz SL, et al. Pediatric sleep questionnaire: prediction of sleep apnea and outcomes. Arch Otolaryngol Head Neck Surg 2007;133(3):216-222. DOI: 10.1001/ archotol.133.3.216.

10. Markovich AN, Gendron MA, Corkum PV. Validating the Childrens Sleep Habit Questionnaire against polysomnography and actigraphy 
in school aged children. Front. Psychiatry 2015;5(188):1-10. DOI: 10.3389/fpsyt.2014.00188.

11. Owens JA, Spirito A, McGuinn M. The childrens sleep habit questionnaire (CSHQ): psychometric properties of a survey instrument for school-aged children. Sleep 2000;2(8):1-9. DOI: 10.1093/sleep/23.8.1d.

12. Skotco BG, Macklin EA, Muselli M, et al. A predictive model for obstructive sleep apnea and Down syndrome. Am J Med Genet 2017;9999:1-8.

13. Vriend JL, Davidson FD, Corkum PV, et al. Sleep quantity and quality in relation to daytime functioning in children. Child Health Care 2012;41(3):204-222. DOI: 10.1080/02739615.2012.6.

14. Asaad T, Abu Ella I, Nasr YA, et al. Sleep Profile in a sample of Egyptian school aged children with depression. Middle East Curr Psychiatry 2014;21(2):63-71. DOI: 10.1097/01.XME.0000444268.42846.3f.

15. Maris M, Verhulst S, Wojciechowski M, et al. Prevalence of obstructive sleep apnea in children with down syndrome. Sleep 2016;39(3): 699-704. DOI: 10.5665/sleep.5554.

16. Whitney DG, Shapiro DN, Peterson MD, et al. Factors associated with depression and anxiety in children with intellectual disabilities. J Intellect Disabil Res 2019;63(5):408-417. DOI: 10.1111/jir.12583.

17. Dekker AD, Sacco S, Carfi A, et al. The behavioral and psychological symptoms of dementia in down syndrome (BPSD-DS) scale: comprehensive assessment of psychopathology in down syndrome. J Alzheimers Dis 2018;63(2):797-820. DOI: 10.3233/JAD170920.
18. Esbensen AJ, Beebe DW, Byars KC, et al. Use of sleep evaluations and treatment in children with down syndrome. J Dev Behav Pediatr 2016;37(8):629-636. DOI: 10.1097/DBP.0000000000000333.

19. Ella $B$, Ghorayeb I, Burbaud $P$, et al. Bruxism in movement disorders: a comprehensive review. J Prosthodont 2017;26(7):599-605. DOI: 10.1111/jopr.12479.

20. Churchill SS, Kieckhefer GM, Bjornson KF, et al. Relationship between sleep disturbance and functional outcomes in daily life habits of children with Down syndrome. Sleep 2015;38(1):61-71. DOI: 10.5665/sleep.4326.

21. Alwhaibi RM, Aldugahishem HM. Factors affecting participation in physical activities in Saudi children with Down Syndrome: mothers' perspective. Disabil Rehabil 2019;41(13):1524-1535. DOI: 10.1080/09638288.2018.1433241.

22. Choi EK, Jung E, Van Riper M, et al. Sleep problems in Korean children with Down syndrome and parental quality of life. J Intellect Disabil Res 2019;63(11):1346-1358. DOI: 10.1111/jir.12675.

23. Ono J, Chishaki A, Ohkusa T, et al. Obstructive sleep apnea-related symptoms in Japanese people with Down syndrome. Nurs Health Sci 2015;17(4):420-425. DOI: 10.1111/nhs.12206.

24. Landry ML, Rompre PH, Manzini C, et al. Reduction of sleep Bruxism using a mandibular advancement device: an experimental controlled study. Int J Prosthodont 2006;19(6):549-556.

25. Simpson R, Oyekan AA, Ehsan Z, et al. Obstructive sleep apnea in patients with Down syndrome: current perspectives. Nat Sci Sleep 2018;10:287-293. DOI: 10.2147/NSS.S154723. 